\title{
Refractory hypertension and sleep apnoea: effect of CPAP on blood pressure and baroreflex
}

\author{
A.G. Logan*, R. Tkacova\# ${ }^{\#}$ S.M. Perlikowski*, R.S. Leung, ${ }^{\#, ~ A . ~ T i s l e r *, ~ J . S . ~ F l o r a s *, ~}$ \\ T.D. Bradley,, ,+
}

\begin{abstract}
Refractory hypertension and sleep apnoea: effect of CPAP on blood pressure and baroreflex. A.G. Logan, R. Tkacova, S.M. Perlikowski, R.S. Leung, A. Tisler, J.S. Floras, T.D. Bradley. (C) ERS Journals Ltd 2003.

ABSTRACT: This study was undertaken to determine whether abolition of obstructive sleep apnoea (OSA) by continuous positive airway pressure (CPAP) could reduce blood pressure (BP) in patients with refractory hypertension.

In 11 refractory hypertensive patients with OSA, the acute effects of CPAP on nocturnal BP were studied during sleep and its longer term effects on 24-h ambulatory BP after 2 months.

During a single night's application, CPAP abolished OSA and reduced systolic BP in stage 2 sleep from $138.3 \pm 6.8$ to $126.0 \pm 6.3 \mathrm{mmHg}$. There was also a trend towards a reduction in average diastolic $B P$ (from $77.7 \pm 4.5$ to $72.9 \pm 4.5$ ). CPAP usage for 2 months was accompanied by an $11.0 \pm 4.4 \mathrm{mmHg}$ reduction in 24-h systolic BP. In addition, both the nocturnal and daytime components of systolic BP fell significantly by 14.4 \pm 4.4 and $9.3 \pm 3.9 \mathrm{mmHg}$, respectively. Diastolic $\mathrm{BP}$ was reduced significantly at night by $7.8 \pm 3.0 \mathrm{mmHg}$.

In patients with refractory hypertension, acute abolition of obstructive sleep apnoea by continuous positive airway pressure reduces nocturnal blood pressure. These data also suggest that continuous positive airway pressure may reduce nocturnal and daytime systolic blood pressure chronically. Randomised trials are needed to confirm the latter results.
\end{abstract}

Eur Respir J 2003; 21: 241-247.

\begin{abstract}
*Samuel Lunenfeld Research Institute, Mount Sinai Hospital, ${ }^{\text {\#Sleep Research }}$ Laboratories of the Toronto Rehabilitation Institute, "Toronto General Hospital/UHN, and ${ }^{+}$Dept of Medicine and the Centre for Sleep and Chronobiology of the University of Toronto, Toronto, ON, Canada.
\end{abstract}

Correspondence: T.D. Bradley, NU 9112, The Toronto General Hospital of the University Health Network, 200 Elizabeth Street, Toronto ON, M5G 2C4, Canada.

Fax: 14163404197

E-mail: douglas.bradley@utoronto.ca

Keywords: Cardiovascular physiology, respiratory physiology, sleep physiology

Received: April 302002

Accepted after revision: October 16 2002
Only a quarter of hypertensive patients in the USA have blood pressure (BP) levels that are well controlled [1]. Hypertension in some of these patients persists despite multiple antihypertensive medications and excellent compliance [2]. In specialised hypertension clinics the prevalence of such refractory hypertension ranges $5-18 \%$ [3]. These patients are at greater risk for stroke, renal insufficiency and morbid cardiovascular events than patients whose BPs are well controlled by medical therapy [4].

Obstructive sleep apnoea (OSA) has recently been identified as an independent risk factor for the development of hypertension $[5,6]$. However, its potential contribution to refractory hypertension has received little attention. Recently, the current authors described a high prevalence of OSA in patients with refractory hypertension [7]. In these patients, repetitive nocturnal OSA-related surges in BP, associated with recurrent hypoxia, arousals from sleep, activation of the sympathetic nervous system and impaired baroreflex sensitivity (BRS) for heart rate, could contribute to the development or progression of hypertension $[8,9]$. In addition, since antihypertensive drugs do not have a significant influence on the severity of OSA $[10,11]$, these pathophysiological mechanisms may go unchecked in such patients, rendering their hypertension relatively resistant to drug therapy.
Systolic hypertension is an independent risk factor for cardiovascular events. Control of systolic hypertension reduces overall cardiovascular morbidity and mortality [12-15]. Consequently, increasing emphasis has been placed on the importance of lowering elevated systolic BP $[16,17]$. However, recent studies indicate that it is more difficult to control systolic than diastolic hypertension even in a specialist clinic [18].

StAESSEN et al. [19] recently reported that night-time systolic BP predicted cardiovascular morbidity and mortality more accurately than daytime systolic BP. In a previous study, the present authors showed that refractory hypertension in patients with OSA is primarily systolic and relatively more pronounced at night [7]. Therefore, nocturnal increases in systolic BP due to OSA may have particularly adverse effects in patients with refractory hypertension. Consequently, it is possible that treatment of coexisting OSA in patients with refractory hypertension could improve nocturnal and perhaps daytime BP control.

The effects of nasal continuous positive airway pressure (CPAP) on BP in patients with OSA have been examined in a number of trials [20-25]. However, the great majority of patients who participated were normotensive and CPAP therapy was associated with either slight or no reductions in daytime BP. It remains to be seen whether treating OSA can lower 
nocturnal and/or daytime BP in patients who remain hypertensive despite optimal medical therapy. It was hypothesised that abolition of OSA by CPAP would acutely reduce surges in BP and improve BRS during sleep in patients with refractory hypertension. Following this acute interventional study, the effects of CPAP on 24-h BP were assessed after 2 months of treatment.

\section{Methods}

\section{Subjects}

All patients with refractory hypertension referred to a specialised hypertension clinic over a 6-month period were invited to participate in the study. Refractory hypertension was defined as a daytime clinic BP of $\geqslant 140 \mathrm{mmHg}$ systolic or $\geqslant 90 \mathrm{mmHg}$ diastolic, despite a stable rational combination of three or more antihypertensive medications, including a diuretic, unless contraindicated, titrated to maximally recommended doses as tolerated [26] for at least 1 month prior to entry into the study. Patients with the "whitecoat" effect were excluded by 24-h ambulatory BP monitoring [27], as were those with a history of noncompliance with drug treatment, use of medications that can raise $\mathrm{BP}$ or interfere with the actions of antihypertensive agents, any secondary form of hypertension including renal insufficiency (defined as a serum creatinine level $\geqslant 150 \mu \mathrm{mol} \cdot \mathrm{L}^{-1}$ ), excess alcohol use or elevated liver enzymes (more than twice the upper limit of normal), haemodynamically significant aortic or mitral valve disease or previously documented OSA. Patients meeting these criteria then underwent overnight polysomnography to determine whether they had OSA (as defined below). Those found to have OSA were invited to receive a 2-month trial of CPAP.

\section{Ambulatory blood pressure monitoring}

Ambulatory BP was monitored for $24 \mathrm{~h}$ using an automated sphygmomanometer (SL-90207; SpaceLabs Medical, Redmond, WA, USA). An appropriate-sized cuff was fitted to the nondominant arm. Patients recorded the times when they retired at night and awoke in the morning. The BP was recorded every 20 min during the $24-h$ period.

\section{Sleep studies}

Polysomnography was performed using standard techniques and scoring criteria for the determination of sleep stages and movement arousals [28-30]. The electrocardiogram was recorded from a precordial lead from which heart rate was derived. Thoracoabdominal movements were measured by a calibrated respiratory inductance plethysmograph (Respitrace; Ambulatory Monitoring Inc., White Plains, NY, USA) [31]. Arterial oxygen saturation $\left(\mathrm{Sa}, \mathrm{O}_{2}\right)$ was measured with an ear oximeter (Biox 3700; Ohmeda, Boulder, CO, USA). Obstructive apnoeas were identified by the absence of, or a $50 \%$ or greater reduction in, tidal volume for at least $10 \mathrm{~s}$ during which there were paradoxical thoracoabdominal movements. The apnoea/ hypopnoea index (AHI) was defined as the number of apnoeas and hypopnoeas per hour of sleep. OSA was defined as at least 10 obstructive apnoeas and hypopnoeas per hour of sleep. In addition to the above, on the acute CPAP intervention night (see below), finger BP was measured continuously by digital photoplethysmography (Finapres 2300 BP Monitor; Ohmeda, Englewood, CO, USA) with patients supine, and the arm and hand secured on a splint and maintained in the horizontal position.

\section{Protocol}

The study protocol was approved by the Human Subjects Review Committee of the University of Toronto (Toronto, ON, Canada). All patients provided written informed consent prior to participation. Patients with refractory hypertension identified in the hypertension clinic underwent a diagnostic overnight polysomnography. Those found to have OSA were invited to participate in the interventional protocol, which consisted of acute and chronic studies. In the acute study, subjects were studied during two consecutive nights in the sleep laboratory. During the first night, CPAP was titrated to the optimal pressure at which apnoeas and hypopnoeas were abolished. On the second night, once the subjects fell asleep, they were studied: 1) while off CPAP for at least $1 \mathrm{~h}$ of stable nonrapid eye movement (non-REM) sleep during the first part of the night (pre-CPAP); and 2) while on CPAP (using the optimal pressure determined during the first night) for $2-3 \mathrm{~h}$ during the second part of the night.

For the chronic study all subjects underwent 24-h ambulatory BP monitoring prior to initiation of CPAP. They were then given CPAP machines and were instructed to use CPAP for at least $6 \mathrm{~h}$ per night at home at the optimum pressure determined during CPAP titration. Two months later a repeat 24-h BP assessment was performed. Compliance with CPAP was assessed at the end of the trial using a time-onpressure recording.

\section{Data analysis}

In the acute study, beat-by-beat BP and heart rate data were analysed during stage-2 sleep using a customised computer program. Variables were averaged over five consecutive obstructive apnoeas and five ventilatory periods between apnoeas. The program also identified sequences of at least three consecutive cardiac cycles during which increases or decreases in systolic BP were accompanied by simultaneous increases or decreases in R-wave to R-wave intervals, respectively, over 7-min periods. Based on these sequences, spontaneous BRS for heart rate was determined as previously described [29, 32]. The analysis of BP, heart rate and BRS was confined to stage- 2 sleep to control 

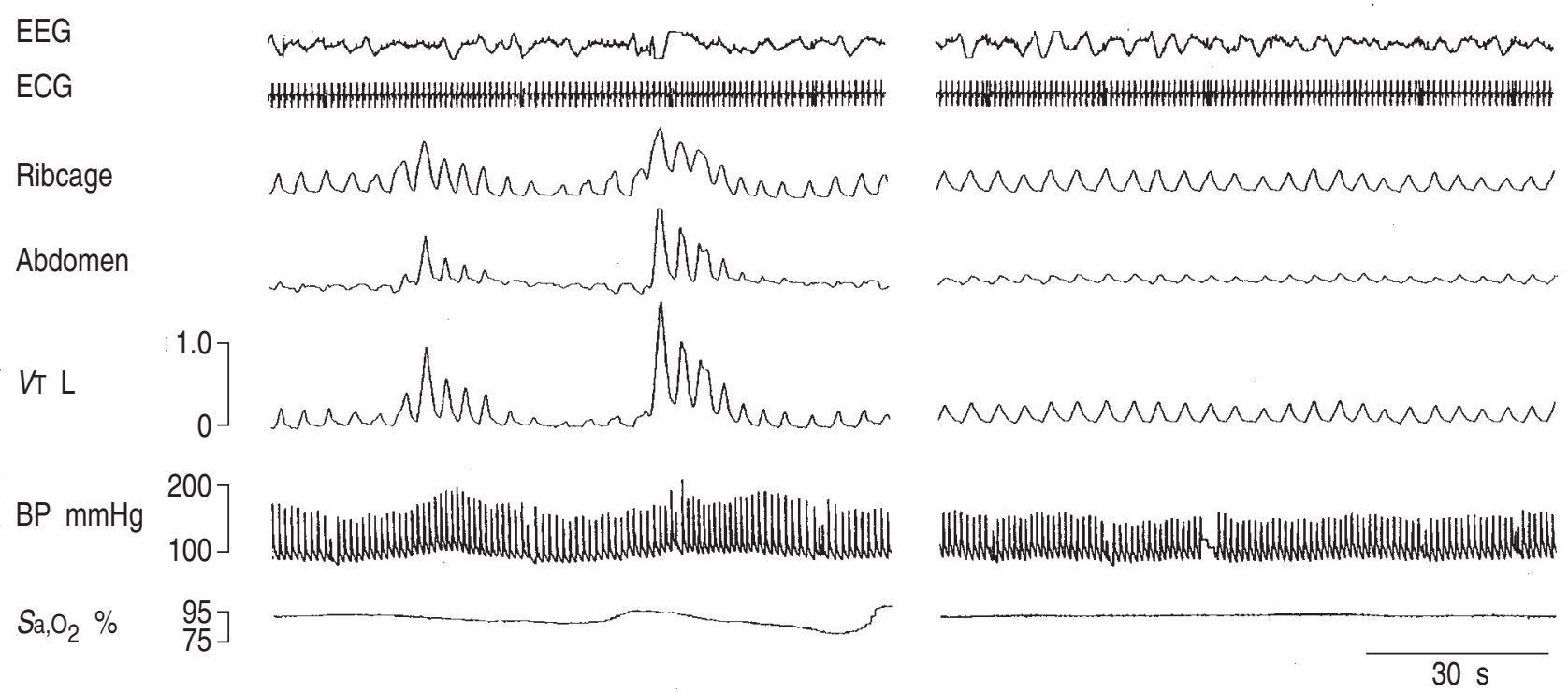

Fig. 1.-A typical polysomnographic recording from a patient with refractory hypertension and obstructive sleep apnoea (OSA) off and then on continuous positive airway pressure (CPAP). Abolition of OSA and improvement in oxyhaemoglobin saturation $\left(\mathrm{Sa}_{\mathrm{a}} \mathrm{O}_{2}\right)$ by $\mathrm{CPAP}$ are accompanied by elimination of the surges in blood pressure (BP). EEG: electroencephalogram; ECG: electrocardiogram; $V \mathrm{~T}$ : tidal volume.

for potential influence of sleep state on the outcome variables.

Paired t-tests were used to compare mean values of variables obtained during the pre-CPAP and CPAP periods of stage-2 sleep during the acute study. Data collected after 2 months of CPAP therapy in the chronic study were compared with baseline by general linear mixed modelling to take into account repeated measures, subject effects and circadian variation in BP [33]. Data are expressed as mean \pm SEM.

\section{Results}

\section{Characteristics of the subjects}

Of 19 patients with refractory hypertension, 16 were found to have OSA. Of these, 11 (10 male and one female) agreed to participate in the acute and chronic CPAP intervention studies. Their mean age was $57 \pm 2$ yrs and their mean body mass index (BMI) was $34.4 \pm 2.4 \mathrm{~kg} \cdot \mathrm{m}^{-2}$. All patients had long-standing hypertension with an average duration of $17 \pm 3$ yrs. They had also been on antihypertensive medications for an average of 16 yrs (range 6-27 yrs). Five subjects had coronary artery disease and two had hypertensive cardiomyopathy. Daytime clinic BP, measured by cuff, was $156 \pm 6$ over $96 \pm 3 \mathrm{mmHg}$. Antihypertensive medication consisted of a diuretic in nine, a calcium-channel blocker in 11 , a $\beta$-adrenergic blocker in eight and an angiotensin-converting enzyme inhibitor in 10 subjects. Subjects had moderately severe OSA, indicated by an AHI of $36.6 \pm 6.6 \mathrm{~h}^{-1}$, frequency of movement arousals of $39.7 \pm 6.0 \cdot \mathrm{h}^{-1}$, and a lowest $\mathrm{Sa}, \mathrm{O}_{2}$ of $81.4 \pm 2.2 \%$.
Acute effects of continuous positive airway pressure during stage-2 sleep

A representative example of the acute effects of CPAP on obstructive apnoeas, $\mathrm{BP}$ and $\mathrm{Sa}_{\mathrm{a}} \mathrm{O}_{2}$ during stage-2 non-REM sleep is illustrated in figure 1. CPAP (mean pressure of $9.2 \pm 0.6 \mathrm{cmH}_{2} \mathrm{O}$ ) significantly reduced both AHI and arousal index $(\mathrm{p}<0.01$ and $\mathrm{p}<0.001$, respectively), and raised the lowest $S_{\mathrm{a}, \mathrm{O}_{2}}(\mathrm{p}<0.0001$; table 1$)$.

Table 2 summarises the acute effects of CPAP on $\mathrm{BP}$, heart rate and BRS. CPAP reduced peak systolic BP and peak pulse BP $(p<0.01$ and $p<0.001$, respectively). Peak diastolic BP did not change significantly. There were significant reductions in average systolic BP $(p<0.05)$, average pulse BP $(p<0.05)$ and average heart rate $(p<0.05)$. The slope of BRS for heart rate increased $(\mathrm{p}<0.05)$. There was also a trend for a reduction in average diastolic $B P(p=0.054)$.

Chronic effects of continuous positive airway pressure on 24-h blood pressure

On average, subjects used CPAP for $4.2 \pm 0.4 \mathrm{~h}$. night $^{-1}$ over the 2 -month study period, during which

Table 1.-Acute effects of continuous positive airway pressure (CPAP) on severity of sleep apnoea

\begin{tabular}{lccl}
\hline & Pre-CPAP & On CPAP & p-value \\
\hline AHI n $\cdot \mathrm{h} \mathrm{sleep}^{-1}$ & $45.3 \pm 10.1$ & $2.2 \pm 0.5$ & $<0.01$ \\
Arousal index n $\cdot \mathrm{h} \mathrm{sleep}^{-1}$ & $37.1 \pm 5.9$ & $9.4 \pm 1.6$ & $<0.001$ \\
Lowest $\mathrm{Sa}_{\mathrm{a}, \mathrm{O}_{2} \%}$ & $83.3 \pm 1.1$ & $92.1 \pm 0.7$ & $<0.0001$ \\
\hline
\end{tabular}

Data are presented as mean \pm SEM. AHI: apnoea/hypopnoea index; $\mathrm{Sa}_{\mathrm{a}} \mathrm{O}_{2}$ : arterial oxygen saturation. 
Table 2.-Acute effects of continuous positive airway pressure (CPAP) on blood pressure (BP) and baroreflex sensitivity (BRS) during stage-2 sleep

Pre-CPAP On CPAP p-value

Peak BPsys mmHg

Peak BPdias $\mathrm{mmHg}$ Peak pulse BP $\mathrm{mmHg}$

Average $\mathrm{BP}$ sys $\mathrm{mmHg}$

Average BPdias $\mathrm{mmHg}$

Average pulse $\mathrm{BP} \mathrm{mmHg}$

Average heart rate beats $\cdot \mathrm{mi}$

Slope of BRS $\mathrm{ms} \cdot \mathrm{mmHg}^{-1}$

\begin{tabular}{|c|c|c|}
\hline $155.2 \pm 9.1$ & $140.4 \pm 7.5$ & $<0$. \\
\hline $89.6 \pm 5.1$ & $74.4 \pm 4.7$ & 0.282 \\
\hline $76.6 \pm 5.8$ & $66.0 \pm 5.2$ & $<0.001$ \\
\hline $138.3 \pm 6.8$ & $126.0 \pm 6.3$ & $<0.05$ \\
\hline $77.7 \pm 4.5$ & $72.9 \pm 4.5$ & 0.054 \\
\hline $60.6 \pm 4.4$ & $52.1 \pm 4.2$ & $<0.05$ \\
\hline $67.1 \pm 4.1$ & $63.3 \pm 4.0$ & $<0.05$ \\
\hline $7.7 \pm 2.1$ & $9.9 \pm 2.5$ & $<0.05$ \\
\hline
\end{tabular}

Data are presented as mean \pm SEM. BPsys: systolic blood pressure; BPdias: diastolic blood pressure.

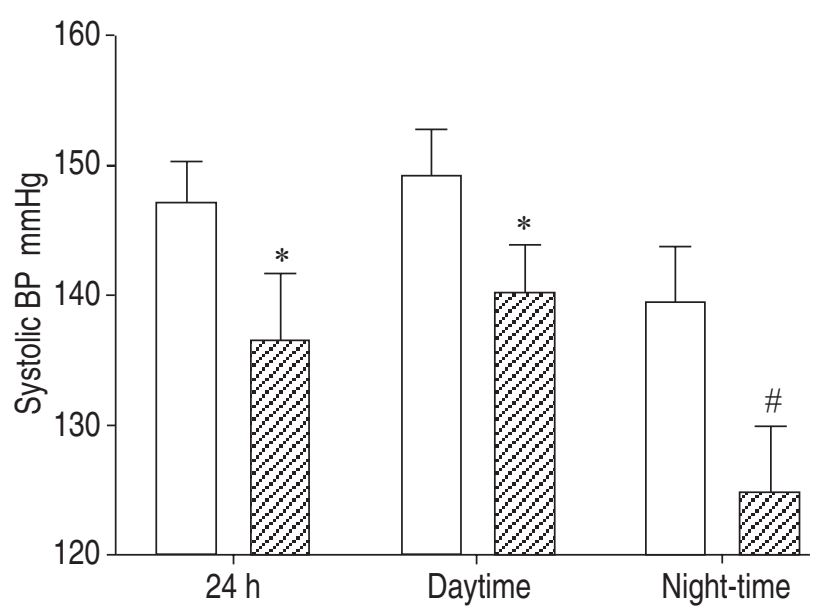

Fig. 2.-Nocturnal continuous positive airway pressure for 2 months $(\mathbb{Z})$ was accompanied by significant reductions in systolic blood pressure (BP) from baseline ( $\square$ ) over $24 \mathrm{~h}$ (from 147.2 \pm 3.3 to $136.2 \pm 3.8 \mathrm{mmHg}$ ), which was due to falls in systolic BP both during the daytime (from $149.9+3.4$ to $140.3+3.7 \mathrm{mmHg}$ ) and at night (from $137.9 \pm 4.2$ to $124.2 \pm 4.5 \mathrm{mmHg}$ ). $*$ : $\mathrm{p}<0.05$ versus baseline; ${ }^{\#}: \mathrm{p}<0.02$ versus baseline

time there was no change in BMI (from 34.4 \pm 2.4 to $34.1 \pm 2.4 \mathrm{~kg} \cdot \mathrm{m}^{-2}$ ). Over this period, 24-h systolic and diastolic BP were significantly reduced due to a treatment effect of $10.5 \pm 4.2 \mathrm{mmHg}(\mathrm{p}<0.05)$ and $5.7 \pm 2.5 \mathrm{mmHg}(\mathrm{p}<0.05)$, respectively. In addition, both the nocturnal and daytime components of systolic BP fell significantly due to treatment effects of $14.4 \pm 4.4 \mathrm{mmHg} \quad(\mathrm{p}<0.005)$ and $9.3 \pm 3.9 \mathrm{mmHg}$ $(\mathrm{p}<0.03)$, respectively (fig. 2). Diastolic BP was reduced significantly at night due to a treatment effect of $7.8 \pm 3.0 \mathrm{mmHg}(\mathrm{p}<0.02$; fig. 3$)$. The treatment effect during the day for diastolic BP was $5.1 \pm 2.6 \mathrm{mmHg}(\mathrm{p}=0.07)$. Heart rate did not change significantly from baseline to 2 months either over $24 \mathrm{~h}\left(72.8 \pm 3.9\right.$ versus $72.7 \pm 3.3$ beats $\left.\cdot \mathrm{min}^{-1}\right)$, during the daytime $\left(75.0 \pm 4.3\right.$ versus $74.4 \pm 3.6$ beats $\left.\cdot \mathrm{min}^{-1}\right)$ or at night $\left(67.0 \pm 3.8\right.$ versus $66.7 \pm 2.8$ beats $\left.\cdot \mathrm{min}^{-1}\right)$.

\section{Discussion}

The present study provides two novel observations on the potential significance of OSA in patients with

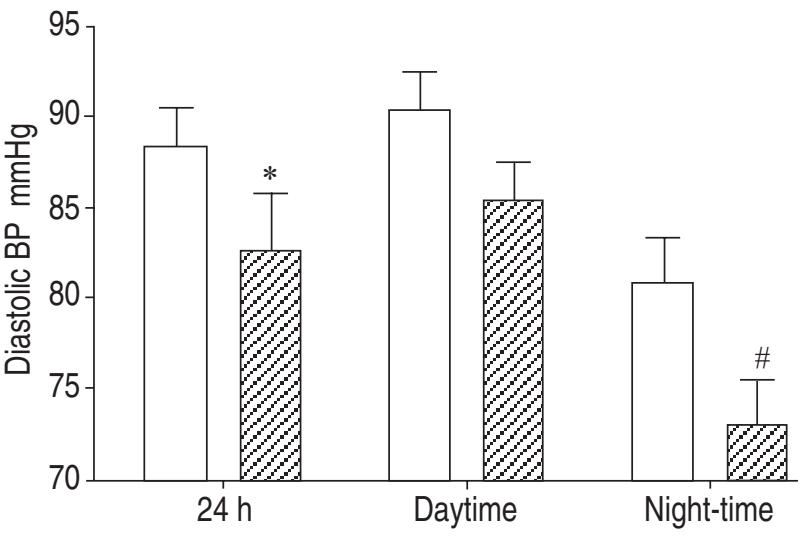

Fig. 3.-Nocturnal continuous positive airway pressure for 2 months $(\mathbb{Z})$ was associated with a reduction in diastolic blood pressure (BP) from baseline ( $\square$ ) both over $24 \mathrm{~h}$ (from $88.3 \pm 2.2$ to $82.6 \pm 3.2 \mathrm{mmHg}$ ) and at night (from $80.8 \pm 2.5$ to $72.9 \pm 2.5 \mathrm{mmHg}$ ). There was also a tendency for daytime diastolic $\mathrm{BP}$ to decrease (from $90.4 \pm 2.0$ to $85.4 \pm 2.0 \mathrm{mmHg}, \mathrm{p}=0.07$ ). *: $\mathrm{p}<0.05$ versus baseline; ${ }^{\#}: \mathrm{p}<0.02$ versus baseline.

refractory hypertension. First, acute abolition of OSA during stage-2 sleep by CPAP reduced systolic BP and increased BRS. Secondly, CPAP therapy for 2 months was accompanied by reductions in nocturnal, daytime and 24-h systolic BP. These results suggest that in patients with refractory hypertension, treating coexisting OSA with CPAP may reduce both nocturnal and daytime BP.

Several factors may play a role in the development of refractory hypertension, including misdiagnosis ("white coat syndrome"), noncompliance with medical therapy, occult secondary causes for hypertension, obesity, confounding effects of medications and other substances, and suboptimal combinations of antihypertensive therapies [2]. With the exception of obesity, these factors were all excluded in these patients. Because obesity itself may have contributed to refractory hypertension, all obese subjects had been counselled to lose weight on numerous occasions prior to entry into the trial, but had failed to do so. Before enrolment, all patients were seen repeatedly, at 2-4 week intervals, by a hypertension specialist, to titrate medications to maximal doses tolerated and to maximise patient compliance with the prescribed medications. Only those patients whose daytime clinic BP exceeded the BP criteria for refractory hypertension [26] despite these efforts were enrolled in the study. Seven of 11 patients studied were obese (BMI $>30 \mathrm{~kg} \cdot \mathrm{m}^{-2}$ ). However, there was no change in their weight over the 2-month study period. Moreover, BP also fell in the nonobese patients.

None of the patients in the present study had sought medical attention for possible sleep apnoea prior to entering the trial. Therefore, it was not possible to determine the duration of their OSA, nor whether it preceded the onset of their hypertension. Nevertheless, two lines of evidence point to a causal relationship between OSA and arterial hypertension. First, in dogs, BrooKs et al. [34] demonstrated that induction of OSA caused sustained daytime elevations of BP that were reversed when OSA was relieved. Secondly, recent large epidemiological studies have 
identified OSA as an independent risk factor for both the presence and development of hypertension [5, 6]. A dose/response association between the frequency of apnoeas and hypopnoeas and degree of elevation in daytime BP has also been described [6]. In addition, the current authors have recently reported an extraordinarily high $(83 \%)$ prevalence of OSA in patients with refractory hypertension [7]. In the present study, reductions in systolic BP during sleep in the acute study, and during both the night and daytime after 2 months of CPAP in the chronic study, provide additional evidence for a causal relationship between OSA and both nocturnal and daytime hypertension.

Normally, at the transition from wakefulness to non-REM sleep, BP falls and BRS increases in association with an increase in parasympathetic and a decrease in sympathetic nervous system activity [35]. However, in OSA, these mechanisms are counteracted by apnoea-related stimuli for sympathetic activation and parasympathetic withdrawal (i.e. repetitive hypoxaemia and arousals) during sleep. These lead to repetitive surges in BP [8] and blunting of BRS for heart rate at the onset of non-REM sleep [9]. The mechanisms that translate these nightly recurrent increases in BP into sustained daytime hypertension may be mediated by central or peripheral upward resetting of sympathetic vasoconstrictor tone, increased activity of circulating vasoactive hormones or abnormalities of vascular endothelial function [36-38].

Several trials have addressed the effects of CPAP on 24-h and daytime BP in OSA patients. However, these trials did not specifically address the effects of treating OSA by CPAP on hypertension, since the great majority of subjects were normotensive [20-24]. Most of these studies reported significant falls in nocturnal $\mathrm{BP}$, but only marginal or no falls in daytime BP. Nevertheless, the two largest randomised trials did show slight but significant reductions in daytime BP (1-2 mmHg) in subjects who complied with CPAP $[20,21]$. Because patients in these studies were either entirely [20] or predominantly normotensive [21], any potential to lower BP in hypertensive patients by treatment of coexistent OSA may have been underestimated. Indeed, PEPPERELl et al. [21], reported that CPAP caused a larger fall in mean 24-h BP $(-6 \mathrm{mmHg})$ in a subset of patients who were on antihypertensive medications than in those who were not $(-2 \mathrm{mmHg})$. This finding suggested that hypertensive patients may have a more pronounced BP-lowering response to CPAP than normotensive subjects. However, the authors did not report how many patients fell into this category, what medications they were on nor what their actual BPs were. The present data are, in general, consistent with those findings, but extend them to patients with uncontrolled hypertension, where reductions in daytime and 24-h systolic BP were more pronounced (9.3 \pm $3.9 \mathrm{mmHg}$ and $10.5 \pm 4.2 \mathrm{mmHg}$, respectively), and were associated with improvements in BRS. Since antihypertensive medications probably caused some lowering of BP in these patients, it is possible that had these medications been discontinued CPAP may have caused an even greater reduction in BP. However, it would have been ethically unjustifiable to discontinue antihypertensive medications in this group of severe hypertensives, many of whom had already suffered complications from their uncontrolled hypertension.

The current protocol differs from previous ones in important ways. First, a well-defined group of refractory hypertensive patients was used, in whom medications were not discontinued during the study period. Secondly, BP in all these patients was managed in a specialised hypertension clinic before inclusion in the study. Thirdly, in contrast to another study [39], reductions in weight did not confound the present results.

What are the potential mechanisms whereby treatment of OSA by CPAP might decrease nocturnal and daytime BP? One likely possibility is that CPAP reduces sympathetically mediated vasoconstriction. These effects have been shown to occur acutely during sleep [8] and to carry over into the daytime after periods of weeks to months [40]. Another possibility is that a downward resetting of the baroreflex threshold and/or an increase in its gain could reset the BP to a lower level and improve its regulation. The present findings of acute increases in BRS, in association with acute reductions in systolic BP and heart rate, provide further evidence that by eliminating OSA, CPAP acutely improves autonomic regulation of heart rate [29]. These improvements were all the more impressive because most patients were on $\beta$-blockers, which could themselves counteract the surges in sympathetic nervous system activity that blunt BRS. While it would have been interesting to examine the influence of CPAP on BRS in these patients while they were off all medications, to do so would have been ethically unjustifiable. Accordingly, these results are clinically relevant since the great majority of patients with severe hypertension will be on antihypertensive medications. Whether these acute nocturnal improvements in BRS are carried over into the daytime and whether they are directly linked to short- or long-term improvements in BP control remains to be addressed in future studies. Since blunted endothelially mediated vasodilation has been reported in OSA, another possible cause of improved BP control with CPAP is that reversal of OSA caused an improvement in vascular endothelial function [37, 38].

Evidence from the Framingham Heart Study indicate that systolic BP is a more robust predictor of cardiovascular risk than diastolic BP [41]. However, controlling systolic BP remains more difficult [18, 19, 42, 43]. Each $10 \mathrm{mmHg}$ increment in ambulatory daytime systolic BP is associated with a $20 \%$ higher cardiovascular risk. Therefore, if reductions in systolic BP of the magnitude observed in this study could be chronically sustained they might improve prognosis in patients with refractory hypertension.

Night-time systolic BP more accurately predicts cardiovascular risk than daytime systolic BP [19]. The reasons for this elevated risk remain poorly understood and have been addressed in only a few studies. In an animal model of hypertension, the best predictor of cardiac hypertrophy was elevated sleeping BP [44]. However, in hypertensive patients with OSA, antihypertensive medications are relatively ineffective at controlling nocturnal systolic BP, even when daytime 
systolic BP is brought under control [45]. These data showed pronounced reductions in nocturnal systolic BP in association with CPAP application for OSA, both acutely and after 2 months of home therapy (12.3 and $14.4 \mathrm{mmHg}$, respectively). The consistency between these acute and chronic reductions in nocturnal systolic BP underscore the clinical significance of the present findings.

A potential problem with this study is the lack of an untreated control group. However, this has little bearing in the acute nocturnal experiments because the fall in nocturnal BP in response to acute application of CPAP was instantaneous, was large in magnitude and occurred simultaneously with abolition of OSA. It could not, therefore, be attributed to a placebo effect. With respect to the chronic part of the study, the two largest randomised placebo-controlled trials of CPAP demonstrated no beneficial placebo effect of CPAP on BP $[20,21]$. Indeed, use of subtherapeutic placebo CPAP in the control group was actually associated with an increase in BP [21]. Moreover, reductions in BP after 2 months of CPAP in this study occurred on a background of unremitting hypertension and neither the types nor dosages of antihypertensive medications were changed during the course of the study. Therefore, it is unlikely that the chronic reductions in $\mathrm{BP}$ observed were due to a placebo effect. In addition, it would be difficult to justify an expensive and time-consuming randomised controlled clinical trial in this highly selected group of refractory hypertensive patients without first obtaining preliminary evidence suggesting the potential for a beneficial effect. Data from this study provide such evidence.

In conclusion, these data demonstrate that continuous positive airway pressure acutely reduces blood pressure and increases baroreflex sensitivity during sleep in patients with refractory hypertension and obstructive sleep apnoea. Although the possibility that a placebo effect or regression to the mean could play a role in the chronic falls observed in systolic blood pressure cannot be ruled out, the data nevertheless suggest that treatment of obstructive sleep apnoea can chronically reduce blood pressure in patients with refractory hypertension. Accordingly, they provide a strong rationale to conduct randomised controlled trials of continuous positive airway pressure in refractory hypertensive patients with obstructive sleep apnoea to assess the validity and generalisability of these findings.

Acknowledgements. This study was supported by operating grants from the Heart and Stroke Foundation of Ontario (grant no. NA 4099) and the Canadian Institutes of Health Research (grant no. MOP-11607). R. Tkacova is supported by a Research Fellowship from Respironics Inc. R. Leung holds a Clinician Scientist Award from the Canadian Institutes of Health Research. A. Tisler was supported by an International Fellowship Training Award from the International Society of Nephrology. J.S. Floras was supported by a Career Investigator Award from the Heart and Stroke Foundation of Ontario. T.D. Bradley is a Senior Scientist of the Canadian Institutes of Health Research.

\section{References}

1. Burt VL, Whelton P, Roccela EJ, et al. Prevalence of hypertension in the US adult population: results form the Third National Health and Nutrition Examination Survey, 1988-1991. Hypertension 1995; 25: 305-313.

2. McAlister FA, Lewanczuk RZ, Teo KK. Resistant hypertension: an overview. Can J Cardiol 1996; 12: 822-828.

3. Setaro JF, Black HR. Refractory hypertension. $N$ Engl J Med 1992; 327: 543-547.

4. Isaksson $\mathrm{H}$, Ostergren J. Prognosis in therapyresistant hypertension. $J$ Int Med 1994; 236: 643-649.

5. Peppard PE, Young T, Palta M, Skatrud J. Prospective study of the association between sleep-disordered breathing and hypertension. N Engl J Med 2000; 342: 1378-1384.

6. Nieto FJ, Young TB, Lind BK, et al. Association of sleep-disordered breathing, sleep apnea, and hypertension in a large community-based study. JAMA 2000; 14: 1829-1836.

7. Logan AG, Perlikowski SM, Mente A. High prevalence of obstructive sleep apnea in drug resistant hypertension. J Hypertension 2001; 19: 2271-2277.

8. Somers VK, Dyken ME, Clary MP, Abboud FM. Sympathetic neural mechanisms in obstructive sleep apnea. J Clin Invest 1995; 96: 1897-1904.

9. Parati G, DiRienzo M, Bonsignore MR, et al. Autonomic cardiac regulation in obstructive sleep apnea syndrome: evidence from spontaneous baroreflex analysis during sleep. J Hypertens 1997; 15: $1621-1626$.

10. Kraiczi H, Hedner J, Peker Y, Grote L. Comparison of atenolol, amlodipine, enalapril, hydrochlorothiazide, and losartan for antihypertensive treatment in patients with obstructive sleep apnea. Am J Respir Crit Care Med 2000; 161: 1423-1428.

11. Weichler U, Herres-Mayer B, Mayer J, Weber K, Hoffman R, Peter JH. Influence of antihypertensive drug therapy on sleep pattern and sleep apnea activity. Cardiology 1991; 78: 124-130.

12. Feldman RD, Campbell N, Larochelle P, et al. 1999 Canadian recommendations for the management of hypertension. Task Force for the Development of the 1999 Canadian Recommendations for the Management of Hypertension. CMAJ 1999; 161: Suppl. 12, S1-S17.

13. Staessen JA, Fagard R, Thijs L, et al. Randomised double-blind comparison of placebo and active treatment for older patients with isolated systolic hypertension. The Systolic Hypertension in Europe (Syst-Eur) Trial Investigators. Lancet 1997; 350: 757-764.

14. Kostis JB, Davis BR, Cutler J, et al. Prevention of heart failure by antihypertensive drug treatment in older persons with isolated systolic hypertension: SHEP Cooperative Research Group. Lancet 1997; 351: $1755-1762$.

15. SHEP Co-operative Research Group. Prevention of stroke by anti-hypertensive drug treatment in older persons with isolated systolic hypertension: final results of the Systolic Hypertension in the Elderly Program (SHEP). JAMA 1991; 265: 3255-3264.

16. Frohlich ED. Recognition of systolic hypertension for hypertension. Hypertension 2000; 35: 1019-1020. 
17. Izzo JL, Levy D, Black HR. Importance of systolic blood pressure in older Americans. Hypertension 2000; 35: 1021-1024.

18. Singer GM, Black HR. How often can HEDIS goals for hypertension control be achieved in a specialist clinic? Am J Hypertens 2000; 13: 111A.

19. Staessen JA, Thijs L, Fagard R, et al. Predicting cardiovascular risk using conventional vs ambulatory blood pressure in older patients with systolic hypertension. JAMA 1999; 282: 539-546.

20. Faccenda JF, MacKay TW, Boon NA, Douglas NJ. Randomized placebo-controlled trial of continuous positive airway pressure on blood pressure in the sleep apnea-hypopnea syndrome. Am J Respir Crit Care Med 2001; 163: 344-348.

21. Pepperell JC, Ramdassing-Dow S, Crosthwaite N, et al. Ambulatory blood pressure after therapeutic and subtherapeutic nasal continuous positive airway pressure for obstructive sleep apnoea: a randomised parallel trial. Lancet 2002; 3359: 204-210.

22. Dimsdale JE, Loredo JS, Profant J. Effect of continuous positive airway pressure on blood pressure. A placebo trial. Hypertension 2000; 35: 144-147.

23. Wilcox I, Grunstein RR, Hedner JA. Effect of nasal continuous positive airway pressure during sleep on 24-hour blood pressure in obstructive sleep apnea. Sleep 1993; 16: 539-544.

24. Suzuki M, Otsuka K, Guilleminault C. Long-term nasal continuous positive airway pressure administration can normalize hypertension in obstructive sleep apnea patients. Sleep 1993; 16: 545-549.

25. Mayer J, Backer H, Brandenburg U, Penzel T, Peter JH, Wichert PV. Blood pressure and sleep apnea: results of long-term nasal continuous positive airway pressure therapy. Cardiology 1991; 79: 84-92.

26. Gifford RW Jr. Resistant hypertension. Introduction and definitions. Hypertension 1988; 11: Suppl. II, 65-66.

27. Pickering TG. Blood pressure measurement and detection of hypertension. Lancet 1994; 344: 31-35.

28. Rechtschaffen A, Kales AA. A manual of standardized terminology, technique and scoring system for sleep stages of human subjects. Los Angeles, UCLA Brain Information Service/Brain Research Institute, 1968.

29. Tkacova R, Dajani HR, Rankin F, Fitzgerald FS, Floras JS, Bradley TD. Continuous positive airway pressure improves baroreflex sensitivity in patients with obstructive sleep apnea and heart failure. J Hypertens 2000; 18: 1257-1262.

30. Tkacova R, Rankin F, Fitzgerald FS, Floras JS, Bradley TD. Effects of continuous positive airway pressure on obstructive sleep apnea and left ventricular afterload in patients with heart failure. Circulation 1998; 98: 2269-2275.

31. Chada TS, Watson H, Birch S, et al. Validation of respiratory inductive plethysmography using different calibration procedures. Am Rev Respir Dis 1982; 125 : 644-649.

32. Bertinieri G, di Rienzo M, Cavalazzi A, Ferrari AU, Pedotti A, Mancia G. A new approach to analysis of the arterial baroreflex. J Hypertens 1985; 3: Suppl. 3, S79-S81.

33. Cnnan A, Laird NM, Slasor P. Using the general linear mixed model to analyse unbalanced repeated measures and longitudinal data. Stat Med 1997; 16: 2349-2380.

34. Brooks D, Horner R, Kozar LF, Render-Teixeira C, Phillipson E. Obstructive sleep apnea as a cause of systemic hypertension: evidence from a canine model. J Clin Invest 1997; 99: 106-109.

35. Pickering GW, Sleight $P$, Smyth HS. The reflex regulation of arterial pressure during sleep in man. J Physiol 1968; 194: 46P-48P.

36. Fletcher EC. The relationship between systemic hypertension and obstructive sleep apnea: facts and theory. Am J Med 1995; 98: 118-128.

37. Carlson JT, Rangemark C, Hedner JA. Attenuated endothelium-dependent vascular relaxation in patients with sleep apnoea. J Hypertens 1996; 14: 577-584.

38. Kato M, Roberts-Thomson P, Phillips BG, et al. Impairment of endothelium-dependent vasodilation of resistance vessels in patients with obstructive sleep apnea. Circulation 2000; 102: 2607-2610.

39. Rauscher H, Formanek D, Popp W, Zwick H. Nasal CPAP and weight loss in hypertensive patients with obstructive sleep apnoea. Thorax 1993; 48: 529-533.

40. Narkiewicz K, Kato M, Phillips BG, Pesek CA, Davison DE, Somers VK. Nocturnal continuous positive airway pressure decreases daytime sympathetic traffic in obstructive sleep apnea. Circulation 1999; 100: 2332-2335.

41. Dustan HP. 50th anniversary historical article: hypertension. J Am Coll Cardiol 1999; 33: 595-597.

42. Black HR. The paradigm has shifted to systolic blood pressure. Hypertension 1999; 34: 386-387.

43. Hansson L, Zanchetti A, Carruthers SG, et al. Effects of intensive blood pressure lowering and low-dose aspirin in patients with hypertension: principal results of the Hypertension Optimal treatment (HOT) randomised trial. Lancet 1998; 351: 1755-1762.

44. Morgan TO, Brunner HR, Aubert JF, Wang Q, Griffiths C, Delbridge L. Cardiac hypertrophy depends upon sleep blood pressure: a study in rats. $J$ Hypertens 2000; 18: 445-451.

45. Pelttari LH, Hietanen EK, Salo TT, Kataja MJ, Kantola IM. Little effect of ordinary antihypertensive therapy on nocturnal high blood pressure in patients with sleep disordered breathing. Am J Hypertens 1998; 11: 272-279. 